\title{
Effect of prenatal EPA and DHA on maternal and cord blood insulin sensitivity: a secondary analysis of the mothers, omega 3, and mental health study
}

Joey A. England ${ }^{1 *}$ D, Joses Jain², Bradley D. Holbrook², Ronald Schrader ${ }^{3}$, Clifford Qualls ${ }^{3}$ and Ellen Mozurkewich ${ }^{2,4}$

\begin{abstract}
Background: We sought to determine whether prenatal supplementation with the omega-3 fatty acids eicosapentaenoic acid (EPA) or docosahexaenoic acid (DHA) would increase markers of insulin sensitivity in maternal or cord blood compared with placebo supplementation. A secondary aim was to evaluate the association of serum EPA and DHA fractions with adiponectin, leptin and the adiponectin:leptin ratio (ALR). We hypothesized that omega-3 fatty acid supplementation would increase markers of insulin sensitivity in maternal and umbilical cord plasma.
\end{abstract}

Methods: We analyzed stored plasma samples collected from a prior 3-arm prospective, double-blinded, randomized controlled trial in which 126 women with singleton pregnancies between 12- and 20-weeks' gestation were randomized to receive: 1) an EPA-rich fish oil supplement, 2) a DHA-rich fish oil supplement, or 3) a soy oil placebo. Maternal venous blood samples were collected at 12-20 weeks gestation (before supplementation) and at 34-36 weeks gestation. At delivery, cord blood was collected. Samples were analyzed using sandwich enzyme-linked immunosorbent assay kits to quantify leptin and adiponectin levels which were utilized to calculate the ALR, a proxy measure for insulin sensitivity.

Results: We found no difference in adiponectin, leptin, and the ALR between the treatment and placebo groups at baseline, after supplementation, or in umbilical cord blood. In regression analyses, higher maternal serum DHA fraction was associated with increased ALR before $(p=0.01)$ and after $(p=0.04)$ DHA supplementation. There was no association of EPA fraction with any measure of insulin sensitivity. Cord blood DHA fraction was significantly associated with cord plasma leptin $(p=0.02)$.

Early pregnancy BMI was significantly associated with maternal leptin levels at baseline and in late pregnancy $(p<0.001)$ and was inversely associated with the ALR $(p<0.001)$. The ALR decreased significantly between the early and late pregnancy visits $(p<0.001)$. Pregnancy weight gain was inversely associated with the ALR $(P .<0.02)$.

Conclusions: EPA- and DHA- rich fish oil supplementation had no effect on plasma markers of insulin sensitivity. However, maternal serum DHA fraction was significantly associated with markers of insulin sensitivity.

Trial registration: https://linicaltrials.gov/, registration number NCT00711971, 7/7/2008.

Keywords: EPA, DHA, Insulin sensitivity, Omega-3 fatty acids

\footnotetext{
* Correspondence: joey.a.england@uth.tmc.edu

'Department of Obstetrics, Gynecology and Reproductive Sciences,

McGovern Medical School, University of Texas Health Science Center at

Houston, 6431 Fannin, MSB 3.286, Houston 77030, USA

Full list of author information is available at the end of the article
}

(c) The Author(s). 2019 Open Access This article is distributed under the terms of the Creative Commons Attribution 4.0 International License (http://creativecommons.org/licenses/by/4.0/), which permits unrestricted use, distribution, and reproduction in any medium, provided you give appropriate credit to the original author(s) and the source, provide a link to the Creative Commons license, and indicate if changes were made. The Creative Commons Public Domain Dedication waiver (http://creativecommons.org/publicdomain/zero/1.0/) applies to the data made available in this article, unless otherwise stated. 


\section{Introduction}

Insulin resistance is associated with several pregnancyassociated morbidities including gestational diabetes, increased birth weight, and increased risk of hypertensive disease [1]. Recent estimated prevalence of gestational diabetes mellitus was estimated as 6\% among women who had a live birth in the United States between 2012 and 2016 [2]. Women with gestational diabetes have an increased risk of developing Type 2 diabetes in later life; a systematic review and meta-analysis involving 20 studies that included 675,455 women found that $12.5 \%$ of women with gestational diabetes developed Type 2 diabetes between 6 weeks after delivery and the end of the various follow-up periods [3]. Risks to the neonate include: hypoglycemia, respiratory distress, cardiac dysfunction and birth injury due to macrosomia [4]. Effects of maternal insulin resistance are not limited to the neonatal timeframe, but may also predispose the child to obesity and Type 2 diabetes [5].

It is not known whether dietary factors during pregnancy may influence the risk for patients to develop insulin resistance and gestational diabetes mellitus. In rodent models, dietary supplementation with omega-3 fatty acid docosahexaenoic acid (DHA) during pregnancy has been shown to be insulin-sensitizing [6]. In several studies in rodents, the omega-3 fatty acids DHA and eicosapentaenoic acid (EPA), have been shown to facilitate formation of insulin sensitizing adipokines such as adiponectin and reduce adipokines associated with insulin resistance $[6,7]$. A recent meta-analysis of trials that evaluated the effect of omega- 3 fatty acid supplementation on glucose control and insulin sensitivity among pregnant women with gestational diabetes mellitus found that supplementation resulted in reduced insulin resistance as measured by the HOMA-IR [8]. However, it is not known whether EPA- and DHA-rich fish oil supplementation would increase markers of insulin sensitivity in pregnant women without diabetes.

Other dietary factors may also influence insulin sensitivity. For example, Vitamin D supplementation has been reported to be insulin-sensitizing in non-pregnant individuals $[9,10]$. Similarly, in a longitudinal study evaluating associations between vitamin $\mathrm{D}$ concentrations and insulin sensitivity during pregnancy, pregnant women who had blood concentrations of vitamin D that were deemed "sufficient" in early gestation had higher blood adiponectin concentrations than pregnant women deemed vitamin D insufficient [11].

The gold standard test to measure insulin resistance and beta-cell function is the Homeostatic Model Assessment (HOMA-IR test) [12]. This was first described in 1985 by Matthews. The approximating equation for insulin resistance used a fasting plasma sample and was delivered by the insulin-glucose product divided by a constant. This model correlates well with estimates using the euglycemic clamp method. However this test requires an overnight fast [13]. In situations in which the HOMA-IR test is impractical, the adipose secreted proteins, adiponectin and leptin, may be used as proxy measures of insulin sensitivity and resistance during pregnancy [14]. In particular, the adiponectin:leptin ratio (ALR) has been shown to be inversely related to the HOMA-IR test [14].

Our study objective was to determine whether maternal EPA- and DHA-rich fish oil supplementation would increase markers of insulin sensitivity in maternal and cord blood plasma compared with placebo supplementation. We hypothesized that maternal EPA- and DHArich fish oil supplementation would increase plasma adiponectin and increase the adiponectin:leptin ratio. We also sought to evaluate the association of serum DHA and EPA fraction as well as other potentially predictive variables available from the parent study, with adiponectin, leptin, and the ALR. We further hypothesized that maternal and umbilical cord DHA serum percentage would be associated with insulin sensitivity in maternal and fetal cord plasma. Our null hypothesis was that maternal fish oil supplementation would lead to no difference in plasma levels of adiponectin and leptin in either maternal or fetal samples compared with controls.

\section{Materials and methods}

We conducted this analysis using maternal plasma samples collected from a prior investigation, "The Mothers, Omega-3, and Mental Health Study," a 3-armed prospective, double-blinded, randomized controlled trial designed to test whether EPA- or DHA-fish oil supplementation would prevent perinatal depressive symptoms among women at risk. The full details of the trial have been previously described $[15,16]$. In brief, participants with singleton pregnancies between 12- and 20-weeks gestation were recruited. Participants were selected based on an elevated risk for depression, defined as a past history of major depressive disorder, a history of postpartum depression, or an Edinburgh Postnatal Depression Scale (EPDS) score between 9 and 19. Exclusion criteria included age $<18$, current major depressive disorder diagnosis, bipolar disorder or schizophrenia, substance abuse disorder, history of bleeding disorder, or clotting disorder requiring anticoagulation $[15,16]$. Potential subjects taking antidepressant medications or omega-3 fatty acid supplements at baseline were excluded from participation in the study. However enrolled participants were allowed to initiate antidepressant medications during the course of the trial, if indicated [ 16].Women who met eligibility criteria and who consented to participate were randomized to one of three arms: 1) an EPA-rich fish oil supplement (1060 mg EPA plus 274 mg DHA), 2) a DHA-rich fish oil 
supplement (900 mg DHA plus $180 \mathrm{mg}$ EPA), or 3) a soy oil placebo. Because the DHA- and EPA-rich fish oil capsules were not identical in appearance, all participants took some placebo capsules (double-dummy design). Full details of the randomization procedure and the supplements are described elsewhere $[15,16]$.

Maternal venous blood samples were collected after a 3 hour fast; baseline samples were collected at the time of study enrollment at 12-20 weeks gestation (visit 1), and again between 34- and 36-weeks' gestation (visit 3). After delivery, a sample of fetal blood was collected from the umbilical cord (visit 4). Samples were centrifuged within $12 \mathrm{~h}$ of collection and plasma aliquots were stored at -70 degrees Celsius.

Stored maternal and umbilical cord plasma samples from participants of the study were analyzed using commercially-available sandwich enzyme-linked immunosorbent assay kits (EMD Millipore, St. Charles, $\mathrm{MO})$ to quantify leptin and adiponectin levels according to the manufacturer's protocols $[17,18]$. The manufacturer's reported within- and between-assay coefficients of variation were 3.3 and $5.7 \%$ respectively for adiponectin and 2.6 and $3.75 \%$ respectively for leptin $[17,18]$. The only variation from the manufacturer's recommended protocol was a dilution of fetal adiponectin samples to $1: 1000$ as opposed to $1: 500$ for maternal samples, based on higher fetal levels of adiponectin. Absorbance of both adiponectin and leptin were measured at $450 \mathrm{~nm}$ and $690 \mathrm{~nm}$ and the results compared. 25-hydroxyvitamin D levels, as well as DHA and EPA fractions in maternal and cord blood serum had been previously assayed $[16,19]$.

ETHICS: The procedures followed in the parent study were approved and conducted in accordance with the institutional review boards of the University of Michigan Medical Center, Ann Arbor, MI, St. Joseph Mercy Hospital in Ypsilanti, MI, and The University of New Mexico Health Sciences Center Human Research Protections Office. All participants gave written informed consent to participate in the study. Participants granted written permission for blood sample analyses for lipids and for inflammatory markers. The trial was registered on $7 / 7 / 2008$ at clinicaltrials.gov: NCT00711971 under the title: "Does Fish Oil Prevent Depression in Pregnancy and Postpartum".

The analyses of de-identified blood samples and data described in this manuscript were approved as an amendment to the parent study ((HUM00004684) by the IRB at the University of Michigan Medical Center. The secondary blood sample analyses described in this manuscript were judged exempt by the University of New Mexico Health Sciences Center Human Research Protections Office (HRPO12-466).
STATISTICS: Demographic variables were compared among three randomized groups (EPA-rich fish oil, DHA-rich fish oil, and placebo) as means and standard deviations if continuous or ordinal scale using 1-way ANOVA and as frequencies using Fisher's exact test. We computed medians, and interquartile ranges for each outcome parameter, adiponectin, leptin and the adiponectin:leptin ratio (ALR). Using raw (unadjusted) data, in order to test whether these parameters changed over time or were different between groups, we performed repeated measures ANOVA with group as grouping factor and visit as repeated factor. The analysis of the ALR was also performed using repeated measures ANOVA with time (enrollment before supplementation, and after supplementation) as a repeated factor and 3 groups as a grouping factor. This was an intent-to-treat analysis in that all available data were used. Outcomes adiponectin, leptin, and constituents of ALR, were analyzed similarly. Because box plot analysis of the data revealed nonnormality, we also analyzed these data after square root transformation. In addition, each outcome after supplementation was analyzed among the 3 groups using linear regression methods (multivariable regression) with predictors being baseline measures of the given outcome and covariates (BMI at enrollment, maternal weight gain, age, and ethnicity).

As an alternative analysis we also computed the change in the maternal adiponectin, leptin, and the adiponectin:leptin ratio, and compared the change among the three treatment groups. One- way ANOVA were followed by Fisher's post hoc $t$ tests. We used ANCOVA to evaluate initiation of antidepressant medications during the trial as a predictor of the change in adiponectin, leptin, and the ALR.

We performed post-hoc analyses of the relationship of adiponectin, leptin, and the ALR with the following predictive variables: serum DHA fraction, serum EPA fraction, plasma fractions and $25-\mathrm{OH}$ vitamin $\mathrm{D}$ concentrations, maternal BMI at study entry, maternal weight gain, and age at study enrollment were performed using multivariable linear regression. The analysis of the effect of maternal weight gain on the outcome measures was adjusted for maternal weight at study entry.

For these exploratory analyses, the three treatment groups and two maternal time points were pooled unless otherwise specified. We calculated the effect of a one unit increase in each of the predictive variables on the ALR using the two-tailed student's $t$ distribution.

We calculated the medians and interquartile ranges for adiponectin, leptin and the ALR in umbilical cord blood. We used raw unadjusted data, as well as square root transformation to evaluate difference according to group assignment using ANOVA. Covariate adjustments were done for birth weight and length of gestation. The 
Table 1 Demographics

\begin{tabular}{|c|c|c|c|c|}
\hline Variable & $\begin{array}{l}\text { EPA-rich fish oil group } \\
N=36\end{array}$ & $\begin{array}{l}\text { DHA-rich fish oil group } \\
N=37\end{array}$ & $\begin{array}{l}\text { Soy oil placebo group } \\
N=41\end{array}$ & Statistical Significance \\
\hline Maternal age at enrollment & $29.9+/-5.2$ & $30.7+/-4.4$ & $30.4+/-5.9$ & $N S^{*}$ \\
\hline White race & 32 & 28 & 34 & $\mathrm{NS}^{\ddagger}$ \\
\hline BMI at enrollment & $28.5+/-6.3$ & $28.5+/-6.7$ & $27.7+/-8.2$ & $N S^{*}$ \\
\hline Gestational age at enrollment (weeks) & $15.9 / 2.7$ & $16.9 / 2.3$ & $16.0 / 2.3$ & $\mathrm{NS}^{*}$ \\
\hline Gravidity & $2.42 / 1.36$ & $2.55 / 1.22$ & $2.41 / 2.02$ & $\mathrm{NS}^{\ddagger}$ \\
\hline Parity & $0.87 / 0.84$ & $1.08 / 0.94$ & $0.85 / 1.20$ & $N S^{\ddagger}$ \\
\hline
\end{tabular}

NS $=$ not significant *1-way ANOVA * Fisher's Exact test.

Note: This table represents the subset of the total cohort with samples available for analysis. The total study demographics have been previously described [16]

association of DHA and EPA fractions, as well as 25-OH vitamin $\mathrm{D}$ concentrations in cord blood with measures of insulin sensitivity were analyzed by multivariable linear regression. $P$-values $\leq 0.05$ were considered statistically significant.

SAMPLE SIZE CONSIDERATIONS: The current study is a secondary analysis of data from a randomized controlled trial for which the primary outcome was depressive symptoms as measured by the Beck Depression Inventory $[15,16]$. The sample size was chosen based on a hypothesized difference in the BDI score. For this secondary analysis, we performed a post- hoc power analysis to determine the power of the current study to detect a difference in maternal and cord blood adiponectin concentrations based on adiponectin values available in the literature. From Luo et al. [20] we assumed that baseline adiponectin level would have a baseline standard deviation of approximately 3 micrograms $/ \mathrm{ml}$. Assuming a modest within subject correlation of 0.7 , change scores from the enrollment (12-20 weeks) to post supplementation (34-36 weeks) should have a standard deviation of approximately 2.3 micrograms $/ \mathrm{ml}$. For this standard deviation the minimum detectable mean difference between 2 groups from a two-sample t-test with significance of 0.05 and power of 0.8 is 1.5 micrograms $/ \mathrm{ml}$ where the sample sizes for the two groups both are 36 .

For the comparisons of adiponectin, leptin, and the ALR in cord blood, we assumed an adiponectin standard deviation of approximately 8.0 micrograms $/ \mathrm{ml}$, based on Luo, et al. [20] For a two sample t test with this standard deviation a sample size of 32 per group gives significance level of $5 \%$ and power of $80 \%$; the minimum detectable difference is 5.7 micrograms $/ \mathrm{ml}$. The proposed analysis should have superior power and a smaller detectable difference.

\section{Results}

There were 126 participants who were randomized, of whom 118 completed the trial. For this secondary analysis, there were 113 plasma samples available at trial entry, 109 samples available post-supplementation (3436 weeks) as well as 98 cord blood samples. The flow of participants and samples is shown in Fig. 1.

The baseline characteristics of the three randomized groups are shown in Table 1. There were no significant differences in the baseline characteristics between the three randomized groups.

Our primary intent-to-treat analysis using repeatedmeasures modeling showed no significant difference in adiponectin, leptin, and the ALR in maternal plasma between the treatment groups and placebo group at baseline or after supplementation. (Table 2). These results were unchanged after square root transformation of the data.

Of interest, for the total cohort adiponectin significantly decreased between enrollment and 33-36 weeks gestation $(\mathrm{p}<0.001)$ while leptin significantly increased during the same time period $(p=0.008)$. The ALR

Table 2 Adipokine values before and after supplementation according to treatment group

\begin{tabular}{|c|c|c|c|c|c|c|c|}
\hline \multirow[t]{2}{*}{ Parameter } & \multirow[t]{2}{*}{ Visit } & \multirow[t]{2}{*}{ EPA } & \multirow[t]{2}{*}{ DHA } & \multirow[t]{2}{*}{ Placebo } & \multicolumn{3}{|c|}{ RM ANOVA $P$ values } \\
\hline & & & & & Group & Visit & Interaction \\
\hline \multirow[t]{2}{*}{ Adiponectin ng/ml } & 1 & $24.15(17.72,29.70)$ & $26.15(14.94,38.04)$ & $25.29(15.56,33.69)$ & 0.83 & $<0.001$ & 0.97 \\
\hline & 3 & $18.69(13.79,22.05)$ & $19.91(12.95,27.90)$ & $18.92(14.20,25.71)$ & & & \\
\hline \multirow[t]{2}{*}{ Leptin ng/ml } & 1 & $27.93(17.38,35.77)$ & $22.04(11.46,34.44)$ & $19.72(15.04,28.34)$ & 0.13 & 0.32 & 0.86 \\
\hline & 3 & $28.70(19.69,47.33)$ & $24.38(15.97,33.34)$ & $23.91(16.97,32.72)$ & & & \\
\hline \multirow[t]{2}{*}{ A:L Ratio } & 1 & $0.83(0.41,1.63)$ & $1.44(0.44,2.32)$ & $1.17(0.52,1.97)$ & 0.60 & $<0.001$ & 0.92 \\
\hline & 3 & $0.54(0.34,0.91)$ & $0.77(0.50,1.54)$ & $0.75(0.36,1.26)$ & & & \\
\hline
\end{tabular}


Table 3 Delta Adiponectin, Leptin, and ALR according to treatment group

\begin{tabular}{|c|c|c|c|c|}
\hline \multirow[t]{2}{*}{ Parameter } & \multirow[t]{2}{*}{ EPA } & \multirow[t]{2}{*}{$\mathrm{DHA}$} & \multirow[t]{2}{*}{ Placebo } & \multirow{2}{*}{$\begin{array}{l}P \text { values } \\
\text { Group }\end{array}$} \\
\hline & & & & \\
\hline $\begin{array}{l}\Delta \text { Adiponectin } \\
\mathrm{ng} / \mathrm{ml}\end{array}$ & $-5.35(-30.0,6.43)$ & $-5.17(-19.92,16.44)$ & $-6.66(-41.10,15.27)$ & 0.75 \\
\hline $\begin{array}{l}\Delta \text { Leptin } \\
\mathrm{ng} / \mathrm{ml}\end{array}$ & $2.83(-27.93,35.03)$ & $-0.05(-40.93,17.60)$ & $3.39(-25.19,19.48)$ & 0.45 \\
\hline$\Delta$ A:L Ratio & $-0.47(-5.47,0.37)$ & $-0.51(-4.16,0.40)$ & $-0.68(-6.11,0.61)$ & 0.68 \\
\hline
\end{tabular}

Note: P-values for an among group difference were computed using 1-way ANOVA. Reported adiponectin concentrations reflect 1:500 dilution

significantly decreased during this same time period $(p<$ 0.001 ) indicating increasing insulin resistance with advancing pregnancy. The magnitude of decrease in the ALR was $31 \%$.

Detailed comparison of change (delta) among the 3 treatment groups found no significant differences among the three groups of delta adiponectin, leptin, or the ALR. (Table 3).

Initiation of antidepressant medications during the trial was not significantly predictive of the ALR $(P=$ 0.13).

We performed regression analyses to explore the relationships between measured serum DHA and EPA fraction at enrollment and after supplementation with measures of insulin sensitivity. Variables included in the model included BMI at enrollment, DHA fraction, EPA fraction, vitamin D concentration, and age at enrollment. After adjusting for BMI and maternal age, higher maternal serum DHA fraction was positively associated with increased ALR before $(p=0.01)$ and after $(p=0.04)$ supplementation. DHA fraction was positively associated with adiponectin after supplementation $(p=0.03)$; there was a non-significant trend towards association of DHA with adiponectin at study enrollment. There was no significant association of EPA with any measure of insulin sensitivity. Vitamin D concentration was also positively associated with adiponectin $(p<0.05)$ at enrollment but was not significantly associated with adiponectin after supplementation.

Early pregnancy BMI was positively associated with maternal leptin levels at baseline and in late pregnancy $(p<0.001)$ and was inversely associated with the ALR $(p<0.001)$. ALR decreased significantly between the early and late pregnancy visit $(\mathrm{p}<0.001)$. After adjusting for baseline weight, maternal weight gain between the early pregnancy visit and the late pregnancy visit was positively associated with plasma leptin $(p<0.01)$ and negatively associated with the ALR $(p<0.02)$.

The effect of a one unit increase in each of the variables of interest on the ALR is shown in Table 4.

There were no differences in adiponectin, leptin, or the ALR in umbilical cord blood according to maternal treatment allocation group. (Table 5) Regression analysis was performed to evaluate the association of EPA fraction, DHA fraction, vitamin D concentration, birth weight, gestational age at delivery, and mode of delivery with adiponectin, leptin and the ALR in umbilical cord blood. There were no significant associations between the variables of interest on cord blood adiponectin or the ALR. However, birth weight and cord blood DHA fraction were significantly associated with serum leptin ( $p=0.02$, both). The association of birth weight with cord blood leptin persisted when controlling for DHA fraction, and the association of DHA fraction with cord blood leptin persisted when controlling for birth weight.

\section{Discussion}

The main finding of our study was that DHA- and EPArich fish oil supplementation had no significant effect on plasma markers of insulin sensitivity, compared to placebo. However, maternal serum DHA fraction and plasma $25-\mathrm{OH}$ vitamin D were significantly associated with markers of insulin sensitivity. Of interest, we found that maternal BMI and weight gain negatively affect insulin sensitivity as measured by the ALR, increased maternal leptin and decreased maternal adiponectin levels.

The findings of our study were similar to that of the Haghiac trial [21], which randomly assigned 49 overweight pregnant women to omega-3 fatty acid supplementation conferring $2 \mathrm{~g}$ DHA plus EPA daily; this study found no difference in adiponectin, leptin, or HOMA-IR between the randomized groups. Similarly, a pilot study of $3.36 \mathrm{~g}$ of omega-3 fatty acid supplementation versus matched placebo among 62 hypertensive male adults

Table 4 Variables predictive of the change (delta) in the ALR

\begin{tabular}{|c|c|c|}
\hline Variable & Estimate & $95 \% \mathrm{Cl}$ \\
\hline Pregnancy Weight Gain (1 kg) & $\begin{array}{l}-0.04 \\
(p<0.02)\end{array}$ & {$[-0.07,-0.007]$} \\
\hline Study Enrollment BMI & $\begin{array}{l}-0.03 \\
(p<0.0001)\end{array}$ & {$[-0.04,-0.02]$} \\
\hline After Supplementation BMI & $\begin{array}{l}-0.02 \\
(p<0.001)\end{array}$ & {$[-0.03,-0.01]$} \\
\hline Study Enrollment DHA (1 unit) & $\begin{array}{l}0.2 \\
(p<0.02)\end{array}$ & {$[0.02,0.17]$} \\
\hline After Supplementation DHA (1 unit) & $\begin{array}{l}0.1 \\
(p<0.02)\end{array}$ & {$[0.003,0.112]$} \\
\hline
\end{tabular}

Note. $P$-values and Confidence Intervals $(\mathrm{Cl})$ were based on two-tailed student's t distribution 
Table 5 Cord blood adipokine values according to treatment group

\begin{tabular}{lllll}
\hline Parameter & EPA & DHA & Placebo & Group \\
\hline Adiponectin ng/ml & $52.07(38.99,70.98)$ & $55.67(38.81,68.34)$ & $52.40(43.42,65.70)$ & 0.93 \\
Leptin $\mathrm{ng} / \mathrm{ml}$ & $9.25(4.60,13.55)$ & $7.98(4.26,19.84)$ & $7.10(3.23,13.71)$ & 0.56 \\
A:L Ratio & $5.85(3.63,11.19)$ & $6.21(2.47,15.07)$ & $8.69(4.03,19.36)$ & 0.27 \\
\hline
\end{tabular}

Note. Cell formats are median (IQR)

found no effect of supplementation on serum adiponectin [22].

Our findings contrast with those of the ComparED study [23], which randomized 154 otherwise healthy obese non-pregnant adults to receive $2.7 \mathrm{~g}$ of EPA or DHA versus corn oil placebo. In that population, the investigators found that DHA, but not EPA supplementation significantly increased serum adiponectin, compared with corn oil placebo [23].

Our exploratory analysis of variables predictive of markers for insulin sensitivity found maternal vitamin D concentrations to be associated with markers of insulin sensitivity, similar to the finding of Benaim, et al. [11]

Similar to our findings, in a Mexican cohort, SolisParedis, et al., found maternal leptin levels to be significantly associated with maternal pregnancy weight gain [24]. A birth cohort study by Logan, et al., also found maternal pregnancy weight gain to be associated with decreased insulin sensitivity [25].

Contrary to our expectation, we found serum DHA fraction to be significantly associated with higher cord blood leptin levels. In other investigations, cord blood leptin has been significantly associated with higher birth weight [26, 27]. Elevated cord blood leptin may confer risk for childhood obesity, through developmental programming [26].

Our study had several strengths and limitations. Strengths of our study included the randomized design as well as the double-blinded nature of the trial. Availability of both maternal blood and umbilical cord blood was also a strength, although our study design allowed for only one post-supplementation assessment of insulin sensitivity, timed at 34-36 weeks gestation. However, our study was limited in the respect that our subjects were selected based on predisposition to depression and may not be representative of the general population of pregnant women. Similarly the sample size for the study was determined based on the unrelated primary outcome of depressive symptoms.

\section{Conclusions}

In conclusion, prenatal EPA- and DHA rich fish oil supplementation had no significant effect on markers of insulin sensitivity during pregnancy. However maternal DHA fraction and 25-OH vitamin D concentration were associated with greater insulin sensitivity in pregnancy.
Maternal BMI and pregnancy weight gain were related to lower insulin sensitivity during pregnancy.

Future studies may include a well-powered randomized controlled trial of maternal DHA and/or vitamin D supplementation in women at risk for insulin resistance in order to augment insulin sensitivity.

\section{Abbreviations}

ALR: Adiponection/leptin ratio; DHA: Docosahexaenoic acid;

EPA: Eicosapentaenoic acid

\section{Acknowledgments}

The authors wish to acknowledge the guidance and assistance provided by Susan Tigert, Research Scientist, at the University of New Mexico Clinical and Translational Science Center (CTSC) T1 Laboratory, where the assays described in this study were performed.

The authors also wish to acknowledge Zora Djuric, PhD, who performed the lipid assays for EPA and DHA fraction that were used in statistical analyses of associations with adiponectin and leptin.

This study was previously presented in poster format at the 62nd Annual Scientific Meeting for the Society for Reproductive Investigation, San Francisco, CA, March 25-28, 2015.

\section{Availability of data and material}

The dataset from the original trial is the property of the University of Michigan, Ann Arbor, which was made available to the University of New Mexico under a formal data sharing agreement. The data generated through the secondary blood sample analysis described in this manuscript are the property of the University of New Mexico Health Sciences Center. The datasets will not be publically available but are available from Dr. Mozurkewich, the senior author, and through data sharing agreements with the University of New Mexico Health Sciences Center, and the University of Michigan, on reasonable request. The plasma specimens described in this manuscript were made available to the investigators at the University of New Mexico under a material transfer agreement. They have been analyzed and destroyed, and as such will not be available for further analyses.

\section{Authors' contributions}

EM, and JE designed the study. JE, JJ, and BH performed the blood sample analyses described in the study. RS and CQ analyzed the data. JE, CQ and EM wrote the paper. All authors read and approved the final manuscript.

\section{Authors' information}

Correspondence: Joey A. England, MD (joey.a.england@uth.tmc.edu), Department of Obstetrics, Gynecology and Reproductive Sciences, McGovern Medical School, University of Texas Health Science Center at Houston, 6431 Fannin, MSB 3.286 Houston TX 77030; office phone: 713-500-7780; cell phone: 210-373-1069.

\section{Funding}

The original trial was supported by the NIH R21 AT004166-03S1 (NCCAM), as well as a University of Michigan Clinical Research Initiatives grant and the University of Michigan General Clinical Research Center (now the Michigan Clinical Research Unit). This project was also supported in part by the National Center for Research Resources and the National Center for Advancing Translational Sciences of the National Institutes of Health through Grant Number 8UL1TR000041, The University of New Mexico Clinical and Translational Science Center. The secondary blood sample analyses were paid for by University of New Mexico Department of Obstetrics and Gynecology 
funds. The Nordic Naturals company, Watsonville, California, supplied the supplements and placebos described in this trial.

The National Center for Complementary and Alternative Medicine, through its review process, had a role in design of the parent study. The funding sources had no role in design of this secondary blood sample analysis, or in the collection, analysis, and interpretation of data or in writing the manuscript.

\section{Ethics approval and consent to participate}

The procedures followed in the original trial were approved and conducted in accordance with the institutional review boards of the University of Michigan Medical Center, Ann Arbor, Ml, study number HUM00004684; St. Joseph Mercy Hospital in Ypsilanti, Ml, and The University of New Mexico Health Sciences Center Human Research Protections Office. All subjects gave written, informed consent to participate in the study. The trial was registered at clinicaltrials.gov: NCT00711971 under the title: "Does Fish Oil Prevent Depression in Pregnancy and Postpartum". The secondary blood sample analysis described in this study was approved by the University of Michigan Medical Center Institutional Review Board, and was determined to be exempt by the University of New Mexico Health Sciences Center Human Research Protections Office.

\section{Consent for publication}

Not applicable.

\section{Competing interests}

The authors declare that they have no competing interests.

\section{Author details}

'Department of Obstetrics, Gynecology and Reproductive Sciences, McGovern Medical School, University of Texas Health Science Center at Houston, 6431 Fannin, MSB 3.286, Houston 77030, USA. ²Department of Obstetrics and Gynecology, University of New Mexico, 915 Camino de Salud NE, MSC10-5580, Albuquerque 87131, USA. ${ }^{3}$ Clinical and Translational Center, University of New Mexico, 915 Camino de Salud NE, MSC08-4635,

Albuquerque, NM 87131, USA. ${ }^{4}$ Department of Obstetrics and Gynecology, University of Michigan, L4001 Women's Hospital, 1500 East Medical Center Drive, Ann Arbor, Ml 48109-0276, USA.

\section{Received: 17 June 2019 Accepted: 14 November 2019} Published online: 29 November 2019

\section{References}

1. Catalano PM. The impact of gestational diabetes and maternal obesity on the mother and her offspring. J Dev Orig Health Dis. 2010;1:208-15.

2. Deputy NP, Kim SY, Conrey EJ, Bullard KM. Prevalence and changes in preexisting diabetes and gestational diabetes among women who had a live birth — United States, 2012-2016. MMWR Morb Mortal Wkly Rep, DOl. 2018;67:1201-7 https://doi.org/10.15585/mmwr.mm6743a2.

3. Bellamy L, Casas JP, Hingorani AD, Williams D. Type 2 diabetes mellitus after gestational diabetes: a systematic review and meta-analysis. Lancet. 2009; 373(9677):1773-9 CrossRefExternal Web Site Icon PubMedExternal Web Site Icon.

4. Mitanchez D, Yzydorczyk C, Simeoni U. What neonatal complications should the pediatrician be aware of in case of maternal gestational diabetes? World J Diabetes. 2015;6:734-43.

5. Dabelea D, Mayer-Davis EJ, Lamichhane AP, D'Agostino RB Jr, Liese AD, Vehik KS, et al. Association of intrauterine exposure to maternal diabetes and obesity with type 2 diabetes in youth: the SEARCH case-control study. Diabetes Care. 2008;31(7):1422-6 CrossRefExternal Web Site Icon PubMedExternal Web Site Icon.

6. Bagley HN, Wang Y, Campbell MS, Yu X, Lane RH, Joss-Moore LA. Maternal docosahexaenoic acid increases adiponectin and normalizes IUGR-induced changes in rat adipose deposition. J Obes. 2013;312153.

7. Itoh M, Suganami T, Satoh N, Tanimoto-Koyama K, Yuan X, Tanaka M, et al. Increased adiponectin secretion by highly purified eicosapentaenoic acid in rodent models of obesity and human obese subjects. Arterioscler Thromb Vasc Biol. 2007;27:1918-25.

8. Zhong N, Wang J. The efficacy of omega-3 fatty acid for gestational diabetes: a meta-analysis of randomized controlled trials. Gynecol
Endocrinol. 2019;35(1):4-9. https://doi.org/10.1080/09513590.2018.1480716 Epub 2018 Oct 16. PMID:30324838.

9. Nimitphong H, Samittarucksa R, Saetung S, Bhirommuang N, Chailurkit LO, Ongphiphadhanakul B. The effect of vitamin D supplementation on metabolic phenotypes in Thais with prediabetes. J Med Assoc Thail. 2015;98: 1169-78.

10. Sepehrmanesh Z, Kolahdooz F, Abedi F, Mazroii N, Assarian A, Asemi Z, et al. Vitamin D supplementation affects the beck depression inventory, insulin resistance, and biomarkers of oxidative stress in patients with major depressive disorder: a randomized, controlled clinical trial. J Nutr. 2016;146:243-8.

11. Benaim C, Cocate PG, de Barros EG, Alves-Santos NH, Figueiredo ACC, Franco-Sena $A B$, et al. Longitudinal association of 25 -hydroxyvitamin $D$ with adipokines and markers of glucose metabolism among Brazilian pregnant women. Br J Nutr. 2019;121(1):42-54. https://doi.org/10.1017/ S0007114518003057 PMID:30588902.

12. Tang Q, Li X, Song P, Xu L. Optimal cut-off values for the homeostasis model assessment of insulin resistance (HOMA-IR) and pre-diabetes screening: developments in research and prospects for the future. Drug Discov Ther. 2015;9:380-5.

13. Vogeser M, Konig D, Frey I, Predel HG, Parhofer KG, Berg A. Fasting serum insulin and the homeostasis model of insulin resistance (HOMA-IR) in the monitoring of lifestyle interventions in obese persons. Clin Biochem. 2007; 40:964-8.

14. Skvarca A, Tomazic M, Blagus R, Krhin B, Janez A. Adiponectin/leptin ratio and insulin resistance in pregnancy. J Int Med Res. 2013;41:123-8.

15. Mozurkewich E, Chilimigras J, Klemens C, Keeton K, Allbaugh L, Hamilton S, et al. The mothers, Omega-3 and mental health study. BMC Pregnancy Childbirth. 2011;11:46

16. Mozurkewich EL, Clinton CM, Chilimigras JL, Hamilton SE, Allbaugh $\sqcup$ Berman DR, et al. The mothers, omega-3, and mental health study: A double-blind, randomized controlled trial. American. J Obstet Gynecol. 2013; 208(313):e1-9.

17. EMD Millipore. Human adiponectin elisa kit. EMD Millipore. Revised 25 May 2012. Available at http://www.millipore.com/userguides/tech1/protocol_ ezhadp-61k. Accessed February 18, 2014.

18. EMD Millipore. Human leptin elisa kit. EMD Millipore. Revised 25 May 2012 Available at http://www.millipore.com/userguides/tech1/proto_ezhl-80sk. Accessed February 18, 2014.

19. Williams JA, Romero VC, Clinton CM, Vazquez DM, Marcus SM, Chilimigras $J$, et al. Vitamin D levels and perinatal depressive symptoms in women at risk: A secondary analysis of the mothers, omega-3, and mental health study. BMC Pregnancy Childbirth. 2016;16:203,016-0988-7.

20. Luo ZC, Nuyt AM, Delvin E, Fraser WD, Julien P, Audibert F, Girard I, et al. Maternal and fetal leptin, adiponectin levels and associations with fetal insulin sensitivity. Obesity (Silver Spring). 2013;21(1):210-6. https://doi.org/10. 1002/oby.20250 PMID: 23505188.

21. Haghiac M, Yang XH, Presley L, Smith S, Dettelback S, Minium J, et al. Dietary omega-3 fatty acid supplementation reduces inflammation in obese pregnant women: a randomized double-blind controlled clinical trial. PLoS One. 2015;10:e0137309.

22. Krantz MJ, Havranek EP, Pereira RI, Beaty B, Mehler PS, Long CS. Effects of omega-3 fatty acids on arterial stiffness in patients with hypertension: $A$ randomized pilot study. J Negat Results Biomed. 2015;14(21):015-0040-x.

23. Allaire J, Couture P, Leclerc M, Charest A, Marin J, Lépine MC, Talbot D, Tchernof A, Lamarche B. A randomized, crossover, head-to-head comparison of eicosapentaenoic acid and docosahexaenoic acid supplementation to reduce inflammation markers in men and women: the Comparing EPA to DHA (ComparED) Study.Allaire J et al. Am J Clin Nutr. 2016104(2):280-287. doi: https://doi.org/10.3945/ajcn.116.131896. Epub 2016 Jun 8.

24. Solis-Paredes M, Espino Y, Sosa S, Estrada-Gutierrez G, Nava-Salazar S, Ortega-Castillo V, Rodriguez-Bosch M, et al. Maternal and fetal lipid and adipokine profiles and their association with obesity. Int J Endocrinol. 2016; 7015626.

25. Logan CA, Bornemann R, Koenig W, Reister F, Walter V, Fantuzzi G, et al. Gestational Weight Gain and Fetal-Maternal Adiponectin, Leptin, and CRP: results of two birth cohorts studies. Sci Rep. 2017;2(7):41847. https://doi.org/ 10.1038/srep41847.

26. Donnelly JM, Lindsay KL, Walsh JM, Horan M, Molloy EJ, McAuliffe FM. Fetal metabolic influences of neonatal anthropometry and adiposity. BMC Pediatr. 201515:175,015-0499-0. 
27. Walsh JM, Byrne J, Mahony RM, Foley ME, McAuliffe FM. Leptin, fetal growth and insulin resistance in non-diabetic pregnancies. Early Hum Dev. 2014;90: $271-4$.

\section{Publisher's Note}

Springer Nature remains neutral with regard to jurisdictional claims in published maps and institutional affiliations.

Ready to submit your research? Choose BMC and benefit from:

- fast, convenient online submission

- thorough peer review by experienced researchers in your field

- rapid publication on acceptance

- support for research data, including large and complex data types

- gold Open Access which fosters wider collaboration and increased citations

- maximum visibility for your research: over $100 \mathrm{M}$ website views per year

At $\mathrm{BMC}$, research is always in progress.

Learn more biomedcentral.com/submissions 